\title{
Characterization of water and a model lipophilic compound in human stratum corneum by NMR spectroscopy and equilibrium sorption
}

\author{
CHANDANA KODIWEERA ${ }^{1,2}$, WAYNE J. ROMONCHUK ${ }^{1,3}$, YUAN YANG ${ }^{4}$, ANNETTE \\ L. BUNGE ${ }^{1}$ \\ ${ }^{1}$ Colorado School of Mines, Chemical and Biological Engineering Department, Golden, \\ CO 80401, USA \\ ${ }^{2}$ Dartmouth Brain Imaging Center, Dartmouth College, Hanover, NH 03755, USA \\ ${ }^{3}$ Lakewood, CO 80228, USA \\ ${ }^{4}$ Colorado School of Mines, Department of Chemistry, Golden, CO 80401, USA
}

Correspondence to:

Annette L. Bunge

Chemical \& Biological Engineering Department

Colorado School of Mines

1500 Illinois Street

Golden, CO 80401 USA

phone: +1-303-273-3722

FAX: +1-303-273-3730

Email: abunge@mines.edu

This article contains supplementary material available from the authors upon request

Abbreviations used: DI, deionized water; PBS, phosphate buffered saline solution; TFMB, 2-(trifluoromethyl) benzonitrile; SC, stratum corneum; ${ }^{1} \mathrm{H}$, proton; ${ }^{19} \mathrm{~F}$, fluorine; NMR, nuclear magnetic resonance 


\section{ABSTRACT}

The stratum corneum (SC) is the outermost skin layer in humans and other mammals and the primary barrier to water loss and environmental exposure to chemicals and microorganisms. It consists of flattened, keratin-filled corneocytes surrounded by well-organized lipid layers. Human SC at varying degrees of hydration with and without addition of a model lipophilic compound, 2-(trifluoromethyl) benzonitrile (TFMB), was studied using proton $\left({ }^{1} \mathrm{H}\right)$ and fluorine $\left({ }^{19} \mathrm{~F}\right)$ nuclear magnetic resonance (NMR) techniques. Proton spectral analyses revealed that water mainly occupies the corneocytes in agreement with prior studies. Observations from ${ }^{19} \mathrm{~F}$ spectral and spin-lattice relaxation time $\left(T_{1}\right)$ analyses showed that TFMB is present primarily in the lipids with small amounts in water, which is located within the corneocytes. This is consistent with TFMB sorption, which was measured in SC with and without lipid extraction. The presence of TFMB within the corneocytes supports the hypothesis that transcellular diffusion of a lipophilic compound like TFMB may contribute to SC permeation.

\section{Keywords}

human stratum corneum (SC), NMR spectroscopy, spin-lattice relaxation time, chemical pathways, lipophilic chemicals, sorption, skin, transdermal drug delivery, extracellular lipid, corneocytes, partition coefficient 


\section{INTRODUCTION}

The thin $(20-40 \mu \mathrm{m})$ outermost layer of human skin, called the stratum corneum (SC) is the main barrier to the transfer of water, chemicals and microorganisms. It is a multiphasic, anisotropic membrane composed of approximately 8-20 layers of flattened, hexagonal shaped, keratinenriched corneocytes $(\sim 1 \mu \mathrm{m}$ thick x $40 \mu \mathrm{m}$ diameter $)$ with highly organized lipid lamellae filling the extracellular space $\left(\sim 0.1 \mu \mathrm{m}\right.$ wide) between the corneocytes. ${ }^{1-4}$ It has been claimed that lipophilic compounds primarily transfer across the SC via the tortuous but continuous extracellular lipoidal pathway around the corneocytes. ${ }^{5-7}$ However, calculations from mathematical models that include physicochemical properties of both the lipid and corneocytes phases in a reasonable representation of the multiphasic SC structure suggest that transcellular, corneocyte-phase transport is a potentially significant contributor, even for compounds that are as lipophilic as testosterone,${ }^{8}$ for which the logarithm of the octanol-water partition coefficient $\left.\left(K_{o / w}\right)=3.32^{9}\right)$. Moreover, it has been observed experimentally that the amount of water, which is almost entirely located inside the corneocytes, affects permeability of lipophilic and hydrophilic compounds, including water. ${ }^{10-12}$

A goal of this investigation was to acquire experimental results that could be used in mathematical models testing the alternate hypotheses that SC permeation of lipophilic compounds is predominantly extracellular or transcellular, and to assess whether the permeation mechanism changes with water content in the SC. Human stratum corneum at varying degrees of hydration was studied with and without addition of the model lipophilic compound, 2-(trifluoromethyl) benzonitrile (TFMB), using nuclear magnetic resonance (NMR) techniques. TFMB was selected because the trifluoromethyl group (Figure 1) increases the NMR signal by 3 fold, which decreases the time to achieve a given signal-to-noise by 9-fold compared to a 
molecule with only one fluorine. ${ }^{13}$ Also, TFMB solubility in the SC is favorable because it is relatively small size (171.1 Daltons), moderately lipophilic (estimated $\log K_{o / w}=2.5^{14}$, with a low melting point $\left(7.5^{\circ} \mathrm{C}\right)$ and reasonably soluble in water $(\sim 0.9 \mathrm{mg} / \mathrm{mL}$, determined in this study).

The self-diffusion of ${ }^{1} \mathrm{H}$ and ${ }^{19} \mathrm{~F}$-TFMB determined using pulsed-field gradient NMR is described in two companion papers. ${ }^{15,16}$ The aim of this study was to collect evidence for identifying which SC compartment is assessed by the NMR self-diffusion measurements (i.e., within the corneocytes or the extracellular lipids). First, we determined the distribution of TFMB between the lipids and corneocytes by measuring equilibrium partitioning of TFMB from aqueous solutions into human SC samples that were and were not treated to extract the extracellular lipids. Next, we obtained ${ }^{1} \mathrm{H}$ and ${ }^{19} \mathrm{~F}$ NMR spectra, and measured ${ }^{19} \mathrm{~F}$ spin-lattice relaxation time of the untreated and delipidized SC samples prepared at varying levels of hydration. Recognizing the anisotropic structure of the SC, orientation of the SC membranes was controlled to be either parallel or perpendicular relative to the external magnetic field. For both the partitioning and NMR experiments, the mass fraction of extracellular lipids removed from the delipidized SC was measured.

Several published studies have examined molecular motion in SC from animals or humans using NMR techniques. Mainly these describe ${ }^{1} \mathrm{H}$ measurements (for example ${ }^{17-25}$ ), although a few have examined deuterium $\left({ }^{2} \mathrm{H}\right)^{24,26}$ or carbon $\left({ }^{13} \mathrm{C}\right)^{27,28}$. To our knowledge no prior NMR studies of SC from humans or animals have investigated the presence of an absorbed exogenous lipophilic compound. Also, except for spectral measurements by Foreman ${ }^{20}$ and selfdiffusion determinations by Packer and Sellwood ${ }^{17}$ and Pieper et al. ${ }^{19}$, the effect of orienting the SC sheets relative to the external magnetic field has not been explored. 
Most ${ }^{1} \mathrm{H}-\mathrm{NMR}$ studies have used time domain techniques to investigate the role of water on the SC structure. For example, Vavasour et al. ${ }^{24}$ identified two distinct components that, on the timescale of $10 \mu \mathrm{s}$, were either motionally restricted or isotropically mobile. The signal from the mobile protons was attributed, based on spin-spin relaxation time $\left(T_{2}\right)$ decay, to primarily water with a smaller contribution from a nonexchangeable, non-aqueous component that was present in even the desiccated sample, and assigned to the extracellular lipids. Increasing water content increased the orientational disorder of the motionally restricted protons, which were ascribed to proteins, predominantly keratin, located within the corneocytes. Similar observations were reported by Packer and Sellwood ${ }^{18}$ for powdered SC from guinea pig footpads and by Laule et al. ${ }^{25}$ for normal and psoriatic human SC, with psoriatic SC exhibiting more mobile lipid than normal SC. Silva et al. ${ }^{21}$ used wideline spectroscopy and 2D relaxation-chemical shift correlation spectra, along with $T_{2}$ and spectral measurements, to study water uptake by the SC. Like the studies listed above, they detected that a fraction of the lipids exist in a fluid state, and that corneocyte swelling with increased water content was associated with increased $T_{2}$ for the liquid-like component but caused no change in mobility of the solid component. Their investigations of water in extracted lipids may not reflect the behavior of lipids within the SC because their lipid preparation method was unlikely to have produced the structure and organization that occurs in SC. ${ }^{2,29-32}$

Only a few ${ }^{1} \mathrm{H}$ spectral measurements in SC have been reported previously. In studies of a single sheet of human SC, Foreman ${ }^{20}$ observed two broad peaks, in which the resonance position of one shifted as orientation of the sample with respect to the applied magnetic field moved from parallel to perpendicular. However, it was subsequently discovered that the orientation-dependent chemical shift was sinusoidal and consistent with the behavior of thin 
planar films mounted with respect to the magnetic field. This led Foreman et al.$^{33}$ to attribute the orientation-dependent chemical shift to a thin water film on the SC surface rather than an effect within the SC. Silva et al. ${ }^{21}$ observed a single liquid-like component in the wideline spectra of the delipidized corneocytes and also in the chemical shift dimension of the $2 \mathrm{D}$ relaxationchemical shift correlation spectrum for untreated SC with $\varphi_{w}=0.14$. The study results presented here expand this small database of ${ }^{1} \mathrm{H}$ spectra to explore the effects of hydration and the presence or absence of lipids while controlling sample orientation relative to the external magnetic field.

\section{EXPERIMENTAL}

\section{Chemicals}

The 2-(trifluoromethyl) benzonitrile (TFMB) from Sigma-Aldrich (Milwaukee, WI) was labeled as $98 \%$ minimum purity and used as received. Methanol and chloroform from Mallinckrodt (Phillipsburg, NJ) and acetonitrile were all HPLC grade. Water was de-ionized (DI) and polished with a Millipore MILLI-Q water system (Bedford, MA). Phosphate buffered saline (PBS, 0.01 $\mathrm{mM}$ ) was prepared in DI water using pre-measured pouches that also contain $0.138 \mathrm{M} \mathrm{NaCl}$ and 0.0027 M KCl from Sigma (P3813, St. Louis, MO). Trypsin (Sigma, St Louis, MO) was from either bovine pancreas (product number T1426, TPCK treated essentially salt free, $\geq 10,000$ BAEE units/mg protein, 12780 units/mg solid) or porcine pancreas (product number T7409, lyophilized powder Type II-S, 1645 BAEE units/mg powder).

\section{SC sample preparation}

Split-thickness human skin (approximately 350- $\mu \mathrm{m}$ thick), collected from the abdomen or back within 24 hours post mortem, was purchased from National Disease Research Interchange (NDRI, Philadelphia, PA) and stored at approximately minus $70^{\circ} \mathrm{C}$ until use. Isolated stratum 
corneum (SC) was prepared by enzymatically digesting the dermis and viable epidermis layers from skin samples cut to $\sim 1 \mathrm{~cm} \mathrm{x} 2 \mathrm{~cm}$ and soaked for about $24 \mathrm{~h}$ at $32^{\circ} \mathrm{C}$ (i.e., normal skin temperature in humans) in deionized (DI) water containing $1 \%$ trypsin. The SC was carefully peeled from the digested skin tissues, thoroughly rinsed with DI water, and spread flat onto a small piece of Teflon ${ }^{\circledR}$ sheet (cut from $1 / 16^{\text {th }}$-inch thick, 12 in x 24 in sheet purchased from Small Parts, Inc., Logansport, IN). The SC was then dried in air and stored in a desiccator containing indicating Drierite ${ }^{\mathrm{TM}}$ for later use. Weights of SC samples required at various steps of the preparation protocol were determined using a semi-microgram balance (Mettler Toledo AX26DR Delta Range, Columbus, $\mathrm{OH}$ ) with a Polonium 210 alpha ionizer to control static electricity.

Delipidized SC samples were prepared by soaking a weighed piece of dry isolated SC in 10-20 mL of a 2:1 v:v chloroform:methanol solution for approximately $24 \mathrm{~h}$ with gentle agitation. ${ }^{34,35}$ The SC sample was then rinsed thoroughly with fresh chloroform:methanol solution, and soaked for another hour in $20 \mathrm{~mL}$ of solution without stirring. After three further quick rinses with fresh solution ( $3 \mathrm{~mL}$ each), the $\mathrm{SC}$ was spread flat onto a small piece of Teflon ${ }^{\circledR}$ sheet and residual solvent was removed by evaporation at room temperature in open air for one day. The sample was weighed after drying in a desiccator with Drierite ${ }^{\mathrm{TM}}$ for at least three days. The mass fraction of extractable lipids in the dry SC $\left(f_{l i p}\right)$ was calculated from the change in the dry sample weight compared to the dry weight before lipid extraction.

Desiccated samples were used directly from the desiccator. Hydrated samples were prepared by soaking weighed pieces of dry SC, either with or without lipid extraction, in DI water at $32^{\circ} \mathrm{C}$. Samples containing TFMB were hydrated in DI water saturated with TFMB. After soaking for $24 \mathrm{~h}$, the SC samples were spread flat onto small Teflon ${ }^{\circledR}$ sheets and allowed to 
partially dry in air before being packed into the NMR tube. Different levels of hydration were achieved by varying the drying time. Water sorption for each sample was determined gravimetrically after packing into the NMR tube and reported in terms of mass of water per mass of hydrated SC sample (i.e., the weight fraction, $w_{w}$ ) from which the volume fraction $\varphi_{w}$ was calculated as

$$
\varphi_{w}=\frac{w_{w} \rho_{s c}}{w_{w} \rho_{s c}+\left(1-w_{w}\right) \rho_{w}}
$$

where the density of water, $\rho_{w}$, is $1.0 \mathrm{~g} / \mathrm{mL}$, and the density of dry SC, $\rho_{S C}$, is assumed to be 1.3 $\mathrm{g} / \mathrm{mL} .^{36}$

\section{NMR sample preparation}

Pieces of glass rod $(\sim 2 \mathrm{~cm}$ tall) were placed into the empty NMR tubes (5 mm OD x 7-inch, $\sim 4.3 \mathrm{~cm}$ ID) to lift the SC samples into the measurement zone of the instrument. Within the SC layers, the corneocytes are aligned with the long dimension in parallel with the layer. Therefore, to examine the SC with respect to the long and short dimensions of the corneocytes, the SC was packed into the NMR tubes in layers so that the long dimension of the corneocytes would be oriented either parallel or perpendicular to the direction of the external magnetic field as illustrated in Figure 2a. For samples in the parallel orientation, approximately four pieces of SC, that had been weighed dry before hydration (typically about $30 \mathrm{mg}$ ), were rolled around a weighed pipette tip to give a cylinder that was $2-3 \mathrm{~cm}$ in length. After weighing to determine $\varphi_{w}$, the sample was inserted into the NMR tube, which was capped and sealed with Parafilm ${ }^{\circledR}$. At the end of the experiment, the sample was removed and weighed again to confirm that it had not changed. 
Samples in the perpendicular orientation were prepared by cutting circles of SC using a 3.8-mm hole punch custom made from a stainless-steel tube. A small stack of SC was created by repeatedly cutting circles without emptying the punch. The punch opening was then held in alignment with the opening of the NMR tube and the stack of SC circles was pushed slowly into the NMR tube using a brass rod, being careful to keep the perpendicular orientation. The process was repeated until the desired quantity of SC had been loaded into the NMR tube, after which it was capped and sealed with Parafilm ${ }^{\circledR}$. At the end of the experiment, the hydrated sample was removed from the NMR tube, quickly weighed and then weighed again after the sample had dried (typically about $15 \mathrm{mg}$ ) in the desiccator to determine $\varphi_{w}$.

Samples in the sealed NMR tubes were equilibrated overnight at $32^{\circ} \mathrm{C}$. Once in the NMR diffusion probe, samples were equilibrated for at least $1 \mathrm{~h}$ at the measurement temperature of $25^{\circ} \mathrm{C}$. The temperature at the position of the sample in the probe was calibrated using the known temperature dependence of the ${ }^{1} \mathrm{H}$ chemical shift separation between the two ${ }^{1} \mathrm{H}$ resonance signals observed for methanol.

\section{NMR measurements}

NMR spectra and spin-lattice relaxation time $\left(T_{1}\right)$ measurements were performed on a Bruker AVANCE III $400 \mathrm{MHz}$ spectrometer equipped with a Bruker Diff 60 diffusion probe at $25^{\circ} \mathrm{C}$. Both ${ }^{1} \mathrm{H}$ and ${ }^{19} \mathrm{~F}$-TFMB spectra are the Fourier transformations of the time domain free induction decays recorded after a $90^{\circ}$ pulse illustrated in Figure $2 \mathrm{~b}$; the pulse width was 5 and $6 \mu \mathrm{s}$, for ${ }^{1} \mathrm{H}$ and ${ }^{19} \mathrm{~F}$-TFMB, respectively. Spectral results are reported relative to references: water for ${ }^{1} \mathrm{H}$ and lithium triflate $\left(\mathrm{CF}_{3} \mathrm{LiO}_{3} \mathrm{~S}\right)$ for ${ }^{19} \mathrm{~F}$. The time domain NMR signal may produce more than one peak in the frequency domain if the interested nucleus presents in different chemical 
environments. This peak separation is called the chemical shift, and it is valuable information in characterizing the tissue compartment location of the ${ }^{1} \mathrm{H}$ or ${ }^{19} \mathrm{~F}$-TFMB.

The spin-lattice relaxation time $\left(T_{1}\right)$ measurements for ${ }^{19}$ F-TFMB were obtained using the inversion recovery pulse sequence shown in Figure $2 \mathrm{c} .{ }^{37}$ Values of $T_{1}$ were calculated by least-squares regression of the recovery magnetization $\left(M_{z}\right)$ measured at 10 evolution time $(\tau)$ values $(0.05,0.25,0.5 \mathrm{~s}, 1,2.5,5,10,25,50$ and $100 \mathrm{~s})$ to Eq. (2):

$$
M_{z}=M_{0}\left[1-2 \exp \left(-\tau / T_{1}\right)\right]
$$

where $M_{0}$ is the equilibrium magnetization.

\section{TFMB sorption and saturation concentration measurements}

Procedures were similar to those described previously. ${ }^{38}$ Equilibrium sorption of TFMB into 2-4 mg weighed pieces of dry SC was measured using PTFE $\left(\right.$ Teflon $^{\circledR}$ ) equilibrium microdialyzers (Harvard Apparatus, Holliston, MA). The two capped 0.5-mL chambers were separated by a nonporous poly(dimethylsiloxane) membrane (Mem-100, approximately $100 \mu \mathrm{m}$ thick) from Membrane Products Corporation (Albany, NY), cut to fit with a size 10 cork borer. After soaking overnight in DI water, the SC sample was added to either water or PBS saturated with TFMB at $32^{\circ} \mathrm{C}$ in one chamber. The other chamber was filled with the same TFMB-saturated solution with excess TFMB added.

The assembled dialyzers were incubated at $32^{\circ} \mathrm{C}$ for approximately $72 \mathrm{~h}$, during which time the weight of the dialyzer was monitored to check for mass loss by evaporation or leakage. Separate experiments confirmed that SC sorption of TFMB in dialyzers equipped with the nonporous membrane (to prevent direct SC contact with excess liquid TFMB) required a longer incubation time to reliably reach equilibrium; i.e., $72 \mathrm{~h}$ compared with $24 \mathrm{~h}$ in cells with porous membranes separating the SC from excess solid chemical ${ }^{38}$. After this, a $100 \mu \mathrm{L}$-sample 
collected from the chamber containing the SC was diluted in $1.5 \mathrm{~mL}$ of acetonitrile. The SC sample was removed and quickly rinsed three times in acetonitrile to remove adhering solution and then extracted into $1.5 \mathrm{~mL}$ of acetonitrile for the delipidized $\mathrm{SC}$ sample or $5 \mathrm{~mL}$ of acetonitrile for the untreated SC sample for 8 hours at room temperature. The SC was then extracted for about 16 and then 8 hours in $1.5 \mathrm{~mL}$ samples of acetonitrile. In most cases, concentrations in the solution from the third extraction were zero and less than $10 \%$ of the chemical uptake was found in the second extraction. A typical experiment consisted of SC samples from a single subject divided into three groups of six cells each to study delipidized SC in PBS and untreated SC in PBS and DI water. In one experiment, 20 cells were divided equally among delipidized and untreated SC from two subjects, all in PBS. Altogether sorption was measured in SC samples from 5 different subjects with measurements repeated in one subject.

The experiments were designed to maintain TFMB saturation of the solution in contact with the skin. However, the concentration was sometimes less than saturation by a small amount (Supporting Information, Figure S1 and Table S4). Therefore, sorption values are reported normalized to the measured concentration of TFMB in the solution in contact with the skin at the end of the equilibration period. Although sorption was determined in hydrated SC (i.e., it was equilibrated with DI water or PBS solution), the results are reported as TFMB concentration per mass of dry SC added to the solution in either untreated or delipidized (treated) form; i.e., $C_{\text {untreat }}$ or $C_{d e l i p}$. The sorbed concentration in the lipid portion of the SC per unit mass of lipid removed by extraction $\left(C_{l i p}\right)$ and the fraction of the sorbed TFMB in the untreated SC in this lipid fraction $\left(f_{T F M B, l i p}\right)$ were calculated by mass balance from the mass of TFMB in the untreated and delipized samples and the mass fraction of lipids $\left(f_{\text {lip }}\right)$ :

$$
C_{\text {lip }}=\frac{1}{f_{\text {lip }}}\left(C_{\text {untreat }}-\left(1-f_{\text {lip }}\right) C_{\text {delip }}\right)
$$




$$
f_{T F M B, l i p}=f_{\text {lip }} \cdot C_{\text {lip }} / C_{\text {untreat }}
$$

The chloroform/methanol treatment extracts only the non-covalently bound lipids leaving lipids that are covalently bound to proteins in the cornified cell envelopes intact. ${ }^{39,40}$ Therefore, $C_{d e l i p}$ represents TFMB uptake into the hydrated corneocytes including the cornified cell envelope and lipids bound to it.

Saturation concentrations at physiological temperature $\left(32^{\circ} \mathrm{C}\right)$ of TFMB in PBS and DI water were determined in equilibrium bottle experiments. TFMB concentrations in samples of the aqueous solutions, collected carefully to avoid the excess TFMB floating on the top of the solution, were quantified by HPLC. Measurements were replicated in 5 or 6 bottles.

Solutions were analyzed for TFMB using a Hewlett Packard 1100 series HPLC system with diode array detection (set at $226 \mathrm{~nm}$ ) equipped with a Zorbax Eclipse XDB-C18 analytical column (Hewlett Packard, $4.6 \mathrm{~mm}$ x $25 \mathrm{~cm})$ and a guard column $(4.6 \mathrm{~mm} \times 12.5 \mathrm{~mm})$ with the same packing. The injection volume was $10 \mu \mathrm{L}$ and the mobile phase (60:40 vol:vol methanol:water) at room temperature (typically about $23^{\circ} \mathrm{C}$ ) was pumped at $1 \mathrm{~mL} \mathrm{~min}^{-1}$. The retention time was $6.2 \mathrm{~min}$ and the run time was $7.5 \mathrm{~min}$.

\section{RESULTS AND DISCUSSION}

\section{Lipid content}

The mass fraction of lipid material extracted by the chloroform/methanol treatment $\left(f_{\text {lip }}\right)$ in the sorption and NMR experiments was $0.210 \pm 0.044$ and $0.255 \pm 0.052$ (mean \pm one standard deviation for these and all results reported below) grams of lipid per gram of dry untreated SC, respectively (Table 1). Although somewhat larger than average $f_{\text {lip }}$ reported in previous studies of human SC (e.g., $0.151 \pm 0.0006$ from Pappinen et al. ${ }^{41}$ for an unspecified number of abdominal 
samples ; $0.162 \pm 0.106$ from Raykar et al. ${ }^{34}$ for 35 skin samples, generally measured in either duplicate or triplicate), the range of the measurements ( 0.111 to 0.287 in the sorption experiments and 0.194 to 0.361 in the NMR experiments) was narrower than that observed in the Raykar et al. study (0.032 to 0.462$)$, which was not restricted to skin from the abdomen or back. If only the 11 samples identified in Raykar et al. as from the abdomen or back are considered, then $f_{\text {lip }}=0.257 \pm 0.122$, which is similar to the results reported here. Elias et al. ${ }^{42}$ reported lipid content as a fraction of wet SC weight (see Tables III and IV in this reference) from the abdomen $(0.068 \pm 0.015)$, which was about twice as large as from the leg $(0.029 \pm 0.012)$. The corresponding $f_{\text {lip }}$ values for dry SC, estimated assuming the water content for hydrated corneocytes $\left(u_{c o r}\right)$ is constant at approximately $3.06 \mathrm{~g}$ of water per $\mathrm{g}$ of dry corneocytes (calculated from $2.75 \mathrm{~g}$ of water in a gram of dry SC with $f_{\text {lip }}=0.10$ as proposed by Nitsche et al. $^{43}$; see Supporting Information for details), are $0.23 \pm 0.06$ for abdomen and $0.11 \pm 0.05$ for leg. Thus, $f_{\text {lip }}$ values reported in Table 1 are consistent with measurements of back and abdominal SC from Raykar et al. and Elias et al. ${ }^{34,42}$, which are larger than $f_{\text {lip }}$ for some other body regions, such as the leg, and also larger than 0.151 from Pappinen et $a .^{41}$ for abdominal SC.

Unlike Raykar et al. ${ }^{34}$, Elias et al. ${ }^{42}$ and this study, Pappinen et al. ${ }^{41}$ rinsed with hexane before trypsinization and chloroform:methanol extraction to remove non-SC lipids ${ }^{44}$ (rinsing with hexane after trypsinization has also been used $^{21,45}$ ). The possible contribution of non-SC lipids to $f_{\text {lip }}$ in the studies without a hexane rinse might be estimated from the abdominal SC results reported in Lampe et al. ${ }^{46}$ Using the extraction procedures described by Elias et al. $(1: 2$ chloroform:methanol), Lampe et al. measured (see Tables 1 and 2 in this reference) an extracted lipid weight fraction of $0.065 \pm 0.01$ (which, based on its similarity with Elias et al. ${ }^{42}$, is likely reported on the basis of hydrated $\mathrm{SC}$, although this was not stated) that contained an average 55.5 
weight \% lipid species now known to not exist in the SC; i.e., free sterols (14.0 \pm 2.2$)$, triglycerides (25.2 \pm 9.2$)$ and nonpolar lipids (16.3 \pm 13.0 for sterol/wax esters, squalene, $n$ alkanes). Assuming that the Elias et al. $\operatorname{study}^{42} f_{\text {lip }}=0.23$ (on a dry SC basis) included a similar proportion of non-SC lipids, then $f_{\text {lip }}$ for only the SC lipids is estimated to be 0.15 (i.e., equal to $0.23 / 1.555)$ in close agreement with Pappinen et al. ${ }^{41}$ We hypothesize therefore that the $f_{\text {lip }}$ values reported here and in Elias et al. ${ }^{42}$, Lampe et al. ${ }^{46}$ and Raykar et al. ${ }^{34}$ probably include a significant quantity of non-SC lipids, which were removed by the hexane rinse in Pappinen et al. $^{41}$

\section{TFMB sorption and saturation concentration measurements}

Measurements of the equilibrium TFMB sorption into SC from five different subjects that were and were not treated to remove the lipid fraction $\left(C_{\text {delip }}\right.$ and $C_{\text {untreat }}$, respectively) are normalized by the concentration of the adjacent aqueous solution, either PBS or DI, and listed in Table 2. The saturation concentration of TFMB in PBS, $0.85 \pm 0.019 \mathrm{mg} / \mathrm{mL}$, is a little smaller $(p=0.13)$ than determined in DI water, $0.88 \pm 0.033 \mathrm{mg} / \mathrm{mL}$, which is consistent with a smaller concentration normalized sorption into untreated SC from DI water than from PBS (although the difference was small and not statistically significant, $p=0.78$ ). Sorption from a saturated solution of TFMB into untreated SC, calculated from the product of concentration normalized sorption and the saturation concentration of the adjacent solution, is the same for DI $(51 \pm 28$ $\mathrm{mg} / \mathrm{g})$ and PBS (54 $\pm 27 \mathrm{mg} / \mathrm{g}$ ) as predicted by thermodynamics for SC samples that are not changed differently by equilibration with PBS and DI.

The calculated concentration in the lipid portion of the SC $\left(C_{l i p}\right)$ relative to the adjacent PBS solution, and the fraction of sorbed TFMB in the lipid fraction of the untreated SC $\left(f_{T F M B, l i p}\right)$ are also listed in Table 2. As expected for a lipophilic compound, most of the TFMB in the 
untreated SC is contained in the lipid fraction $(\sim 95 \%)$ that is extracted by the solvent treatment. The concentration of TFMB in the lipids equilibrated with PBS $(247 \mathrm{mg} / \mathrm{g}$, calculated from $C_{l i p} / C_{P B S}$ multiplied by saturation in PBS) is large; at this concentration, TFMB is estimated to occupy approximately 20 volume percent of the TFMB-saturated lipids accounting for the differences in TFMB and lipid densities.

It has been assumed with good evidence that the solute concentration of water within SC samples at equilibrium with aqueous solutions is the same as the aqueous solution. ${ }^{34,43}$ Therefore, sorption to the non-aqueous component of the corneocytes (i.e., proteins and the cell envelope and its covalently bound lipids) relative to the concentration of the adjacent aqueous solution based on mass ratio concentrations, designated $P C_{p r o / w}$ by Nitsche et al. ${ }^{43}$, is related to $C_{d e l i p}$ and the water content of dry corneocytes $\left(u_{c o r}\right)$ as:

$$
P C_{p r o / w}=\frac{C_{d e l i p}}{C_{P B S}}-u_{c o r}
$$

recognizing that the mass of dry corneocytes is equivalent to the mass of dry delipidized SC. Given $C_{\text {delip }} / C_{P B S}=3.97$ and $u_{\text {cor }}=3.06 \mathrm{~g}$ water/g dry corneocytes (described above), on average about $25 \%$ of the TFMB in the hydrated corneocytes is associated with the non-aqueous component compared with $75 \%$ in the water, although the distribution between water and the non-aqueous component of the corneocytes is likely to be variable as indicated by the large standard deviation of the $C_{d e l i p} / C_{P B S}$ measurements. From sorption measurements alone, the presence of TFMB in both the aqueous and the non-aqueous components of the corneocytes is implied but not proven.

The results listed in Table 2 are also presented in terms of common partition coefficients for the SC $\left(K_{S C / w}\right)$, lipids within the SC $\left(K_{l i p / w}\right)$, and the SC corneocytes compared to water $\left(K_{c o r / w}\right)$, which are defined as the equilibrium mass of solute in $j$ per hydrated volume of $j$ (where 
$j$ is the entire SC, the lipids alone, and the corneocytes alone, respectively) divided by the mass of solute per volume in the adjacent aqueous solution. ${ }^{43}$ These partition coefficients were calculated for samples equilibrated with PBS as described by Eqs. (6)- (8)

$$
\begin{aligned}
& K_{S C / w}=\frac{C_{\text {untreat }}}{C_{P B S}} \frac{\rho_{S C, d r y / w e t}}{\rho_{w}} \\
& K_{l i p / w}=\frac{C_{l i p}}{C_{P B S}} \frac{\rho_{l i p}}{\rho_{w}} \\
& K_{c o r / w}=\frac{C_{\text {delip }}}{C_{P B S}} \frac{\rho_{c o r, d r y / w e t}}{\rho_{w}}
\end{aligned}
$$

in which $\rho_{w}$ is the density of water, $\rho_{\text {lip }}$ is the density of lipids (reasonably estimated as 0.9 $\mathrm{g} / \mathrm{mL}^{43}$ ), and $\rho_{\text {pro }}$ is the average density of the non-aqueous components of the corneocytes, assumed to be approximately $1.37 \mathrm{~g} / \mathrm{mL}^{43}$ Two parameters, $\rho_{c o r, d r y / w e t}$ and $\rho_{S C, d r y / w e t}$, which are not true densities, specify the mass of dry $j$ per unit volume of hydrated (wet) $j$, where $j=$ cor (for corneocytes; i.e., the delipized SC) or SC, calculated as:

$$
\begin{aligned}
& \frac{1}{\rho_{c o r, d r y / w e t}}=\frac{1}{\rho_{p r o}}+\frac{u_{c o r}}{\rho_{w}} \\
& \frac{1}{\rho_{S C, d r y / w e t}}=\frac{f_{l i p}}{\rho_{l i p}}+\frac{1-f_{l i p}}{\rho_{p r o}}+\frac{u_{c o r}\left(1-f_{l i p}\right)}{\rho_{w}}
\end{aligned}
$$

The resulting values are $\rho_{\text {cor,dry/wet }}=0.264 \mathrm{~g}$ of dry corneocytes $/ \mathrm{mL}$ of hydrated corneocytes (recognizing that delipidized SC is the same as corneocytes) and $\rho_{S C, d r y / w e t}=0.310 \mathrm{~g}$ of dry $\mathrm{SC} / \mathrm{mL}$ of hydrated SC for the average $f_{\text {lip }}=0.21$ of the samples measured in Table 2 . For $f_{\text {lip }}=$ 0.15 (as reported by Pappinen et al. ${ }^{41}$ for SC rinsed with hexane to remove non-SC lipids), $\rho_{S C, d r y / w e t}=0.295 \mathrm{~g} / \mathrm{mL}$. Note that the calculation of $\varphi_{w}$ specified by Eq. (1) uses $\rho_{S C}=1.30 \mathrm{~g} / \mathrm{mL}$, which is calculated using Eq. (9) with $u_{c o r}=0$ and $f_{\text {lip }}=0.1$. The slightly smaller value calculated 
for $\rho_{S C}\left(1.24\right.$ or $1.27 \mathrm{~g} / \mathrm{mL}$ at $f_{\text {lip }}=0.21$ or 0.15$)$ was not used in the estimate of $\varphi_{w}$ for the hydrated SC samples (see Table S2 and its discussion in the Supporting Information).

Representative examples of published equations for predicting partition coefficients of the SC with and without separation into lipid and non-lipid components are presented in Table 3. ${ }^{43,47-53}$ Overall, the measured $K_{S C / w}=19.7$ (assuming $f_{\text {lip }}=0.21$ or 18.7 for $f_{\text {lip }}=0.15$ ) is not too different from the predictions, which range from 9 to 71 . The differences between measurements and predictions are larger for the individual lipid and corneocytes components but compensating. Compared to the TFMB measurements, the predictions are about 6-fold smaller for $K_{l i p / w}$ and 7fold larger for $K_{c o r / w}$ (due to the 25-fold larger prediction for $P C_{p r o / w}$ ), which results in a predicted $K_{S C / w}$ that is about half of the experimental value.

\section{${ }^{1} \mathrm{H}$ spectra}

The ${ }^{1} \mathrm{H}$ spectra for desiccated and highly hydrated $\left(\varphi_{w}=0.84\right)$ SC samples are compared with DI water in Figure 3a. In these measurements, the SC samples were oriented with the long dimension of the corneocytes parallel to the direction of the external magnetic field. The spectrum for highly hydrated SC (spectrum A) exhibits a single peak, which is consistent with the ${ }^{1} \mathrm{H}$ spectrum for bulk water (spectrum D), and also keratin. As shown in Figure $3 \mathrm{~b}$, the ${ }^{1} \mathrm{H}$ spectra for hydrated and desiccated preparations of human fingernail clippings, which are almost entirely keratin ${ }^{54}$, align with water, making contributions from water and keratin indistinguishable. However, the presence of water is indicated by the increasing peak area (height) at fixed keratin mass with increasing water content.

In the desiccated untreated SC sample (spectrum C), the water/keratin peak shrinks significantly, and a second peak appears at approximately $3.5 \mathrm{ppm}$, which matches the $3.5 \mathrm{ppm}$ chemical shift observed for protons associated with water and fat in biological tissues. ${ }^{55}$ In the 
desiccated lipid extracted sample (spectrum B), the second peak is smaller but still evident because the chloroform/methanol treatment extracts only the non-covalently bound lipids leaving lipids that are covalently bound to proteins in the cornified cell envelopes intact. ${ }^{39,40}$ The association of the second peak with the SC lipids is consistent with it only being visible in samples containing little water and its area decreasing when the lipids were extracted.

Desiccated samples exhibit peaks with broader spectral linewidth compared with hydrated samples (see spectra B and C versus A), which is similar to a solid sample compared with a liquid sample. Hydrated samples contain liquid (unbound) water inside corneocytes, while water in desiccated samples is bound. Spectral linewidths in liquids are narrow due to averaging of anisotropic interactions (e.g., dipolar coupling) by rapid isotropic molecular motion. Reduced mobility of the bound ${ }^{1} \mathrm{H}$ in dry SC samples can cause shorter relaxation times and/or incomplete averaging of spatially anisotropic magnetic interactions, both of which would lead to broader spectral linewidths. ${ }^{13}$ The spectral differences represented in Figure 3 for hydrated and desiccated SC are consistent with the observation by Packer and Sellwood that adding water to dry SC mobilizes an increasing fraction of the nonaqueous, nonexchangeable ${ }^{1} \mathrm{H}$ to a maximum of about $40 \%$, which remains constant for $\varphi_{w}>0.3$. Based on the results presented in Figure 3, NMR determinations of ${ }^{1} \mathrm{H}$ self-diffusion in hydrated SC samples, which will be presented separately, ${ }^{15}$ reflect self-diffusion of water within the keratin filled corneocytes.

\section{${ }^{19} \mathrm{~F}$ spectra}

The spectra for ${ }^{19}$ F-TFMB in highly hydrated SC with and without lipids extracted and oriented parallel to the external magnetic field are compared in Figure 4 with spectra for ${ }^{19}$ F-TFMB in water, neat TFMB and 1-octanol, representing approximately the SC lipids. ${ }^{34}$ The spectrum for TFMB in water exhibits a single peak at $-5.3 \mathrm{ppm}$, which shifts to the right when TFMB is in 1- 
octanol (-5.8 ppm) or neat TFMB (-6.3 ppm). Likewise, only one peak is observed in the spectrum from SC without lipid extraction (spectrum A), which characterizes TFMB dissolved mainly in the SC lipids. This peak aligns approximately with the peaks in octanol and neat TFMB (spectrum C and D).

Two features are evident in the spectrum from the lipid extracted SC (spectrum B). The peak on the right aligns with the peak in the SC sample without lipid extraction (compare spectra A and B), which is consistent with TFMB in the small amount of SC lipids left by the lipid extraction treatment. The magnitude of the TFMB in lipid peak of the lipid extracted sample is small enough for a shoulder to appear on the left side, which is consistent with a second peak that aligns with the TFMB peak in water (compare spectra B and C). The areas under the left and right peaks are proportional to the TFMB mass in the water and lipid environments. Thus, the spectrum for the lipid extracted SC complements the TFMB sorption measurements, showing that TFMB is present in water as well as the lipids. Most, if not all of this water is located within the corneocytes. The volume of water that might be present in the space between the corneocytes after extracellular lipid extraction would be small compared to the water volume inside the corneocytes. ${ }^{31}$ Because the transcellular pathway through the corneocytes is significantly shorter than the tortuous extracellular pathway around the corneocytes, even a small TFMB concentration inside the corneocytes may be sufficient for the transcellular pathway to contribute significantly or even dominate..$^{8,10,11}$

Spectra are shown in Figure 5 for SC oriented either parallel or perpendicular to the external magnetic field. Consistent with the hypothesis that TFMB is absorbed primarily in the lipids, the single peak in samples oriented parallel to the external magnetic field was unaffected by the level of hydration, remaining at $-6.1 \pm 0.1 \mathrm{ppm}$ (compare spectra A and B). In the 
perpendicular orientation, the ${ }^{19}$ F-TFMB peak was much broader than observed in the parallel orientation, although its position did not change (compare spectra A and C). Moreover, the peak width for SC in the perpendicular orientation decreased significantly without changing position when the lipids were extracted (compare spectra $\mathrm{C}$ and $\mathrm{D}$ ). The different hydration levels of the two perpendicular samples are unlikely to have affected the spectra given that TFMB is primarily present in the lipids, which has little water solubility, and the similarity of the spectra for the parallel oriented samples at different hydration levels (see spectra A and B).

Spectral linewidth broadening can arise from lower spin-spin relaxation (transverse relation) time $\left(T_{2}\right)$. Another possibility is that molecular orientation of TFMB relative to the external magnetic field direction was different in SC samples prepared in the perpendicular and parallel orientations, which led to differences in the averaging of spatially anisotropic magnetic interactions, such as chemical shift anisotropy and/or dipolar couplings. The layers of lipid molecules in normal SC are organized on an orthorhombic or hexagonal lattice, which greatly restrict their lateral movement while allowing free rotation around the lipid chain axis. ${ }^{56}$ The planes of these lipid layers are oriented parallel to the corneocytes surface. ${ }^{57,58}$ Because the corneocytes are highly asymmetric $(20-40 \mu \mathrm{m}$ in diameter but only $\sim 1 \mu \mathrm{m}$ tall), in SC samples oriented parallel to the external magnetic field direction, the corneocyte surfaces, along with the planes of the lipid layers, are also oriented predominantly parallel to the external field direction. By the same reasoning, the planes of the lipid layers are oriented predominantly perpendicular to the external field direction for SC in the perpendicular orientation (see cartoon in Figure 5). Also, the amount of TFMB, which is shaped like a circular disk (see Figure 1) packed into the lipid layers is large (estimated above as $\sim 20$ percent of the lipid volume). One can imagine that 
the almost solid-like lipid layers could encourage the large number of TFMB molecules into preferential orientations with asymmetric restrictions on their free motion.

Magnetic field inhomogeneity within the sample can cause spectral linewidth broadening. There is the possibility that magnetic field inhomogeneity was greater for the smaller perpendicular samples (approximately $15 \mathrm{mg}$ of dry SC stacked to a height of about $8 \mathrm{~mm}$ in the perpendicular orientation compared with $30 \mathrm{mg}$ of dry SC rolled to a height of $30-40 \mathrm{~mm}$ in the parallel orientation; see Figure 2a), due to less perfect shimming on the smaller samples. However, the effect of imperfect shimming would most likely be too small to explain the magnitude of the differences observed between the perpendicular and parallel oriented samples. ${ }^{59}$

For the delipidized and untreated samples oriented perpendicular to the external magnetic field, the only difference, other than the level of hydration, is that the delipidized sample has a single lipid layer covalently bound to proteins in the cornified cell envelope instead of stacked multiple lipid bilayers that occupy the extracellular space in the untreated sample. Differences in magnetic inhomogeneity related to sample size should not be a factor because the SC mass in the two samples was similar, although the mass of lipids and, consequently the mass of TFMB, was much smaller in the delipidized sample. One explanation for the decreased spectral linewidth of the delipidized sample is that the single lipid layer covalently bound to the corneocytes cell envelope is less restrictive of TFMB motion compared with the multiple layers in the untreated sample.

Overall, the results shown in Figures 4 and 5 are consistent with all ${ }^{19} \mathrm{~F}$-spectra measured in this study (see Supporting Information, Figures S3 and S4) and the hypothesis that nearly all of the absorbed TFMB was located in the SC lipids unless the quantity of lipids was significantly reduced (i.e., lipids were extracted), and the quantity of water in the corneocytes was large 
(although the requirement of high hydration was not tested). Therefore, the determinations of ${ }^{19} \mathrm{~F}-\mathrm{TFMB}$ diffusion collected in a companion study described separately ${ }^{16}$ are expected to reflect ${ }^{19}$ F-TFMB self-diffusion within the SC lipids.

\section{${ }^{19} \mathrm{~F}$ - Spin-Lattice relaxation times $\left(T_{1}\right)$}

The $T_{1}$ values determined as a function of hydration are presented in Figure $6 \mathrm{a}$ for ${ }^{19} \mathrm{~F}-\mathrm{TFMB}$ in SC oriented either parallel or perpendicular to the external magnetic field and with or without lipids extracted. For a given sample type and orientation, $T_{1}$ did not change with hydration. This is consistent with the hypothesis that the NMR measurements are detecting ${ }^{19} \mathrm{~F}$-TFMB primarily within the SC lipids, which are unaffected by variation in water content within the corneocytes. The average $T_{1}$ values for SC in both orientations and with or without lipids extracted are compared in Figure $6 \mathrm{~b}$ with TFMB in itself, 1-octanol, or water. Individual measurements are indicated with the symbols. The bar height corresponds to the average of measurements at all hydration levels, except for two measurements considered outliers (designated as X), which were excluded from the average. For the parallel oriented samples, lipid extraction reduced $T_{1}$; i.e., energy transfer from ${ }^{19} \mathrm{~F}$ nuclear spins to the SC is more efficient after extracting lipids. Possibly, constraints to some of the rotational, vibrational or translational motions of ${ }^{19} \mathrm{~F}$ on the TFMB molecule are reduced in the single covalently bound lipid layer left by extracting the unbound lipid bilayers. Orientation relative to the external magnetic field appears to have had little effect in samples without extraction, although more perpendicular data are needed to demonstrate this conclusively; data from the two samples oriented perpendicular to the external magnetic field were noisy and may not be reliable. Overall, $T_{1}$ values for TFMB in SC were more similar to $T_{1}$ for TFMB in itself or in 1-octanol than in water, which was larger by more than 2-fold. Thus, 
TFMB is located in an environment that is more like octanol or TFMB than water, which is consistent with its presence mainly in lipids.

\section{CONCLUSION}

On average $20-25 \%$ of the dry SC mass was extracted by the chloroform/methanol treatment, which is consistent with previous measurements of SC from the back or abdomen after accounting for difference in hydration, ${ }^{34,42,46}$ but larger than the $15 \%$ value determined in abdominal SC that was rinsed before lipid extraction with hexane to remove non-SC lipids. ${ }^{41}$ As expected for a lipophilic compound, and consistent with the ${ }^{19}$ F NMR spectral observations, most of the TFMB sorbed into the untreated SC is contained in the extractable lipids ( 95\%). At equilibrium with TFMB-saturated PBS, the lipid concentration is large $(250 \mathrm{mg} / \mathrm{g})$ enough to occupy an estimated 20 percent of the lipid volume. Within the delipidized SC, the distribution of TFMB between the aqueous and the non-aqueous components cannot be distinguished from the sorption measurements. However, the expected presence of TFMB in water is evident in ${ }^{19} \mathrm{~F}$ spectra of hydrated samples in the parallel orientation. The measured amount of TFMB sorption into the untreated SC is predicted reasonably well by several published correlations using an estimated value for $K_{o / w}$.

The hypothesis that water is primarily located within the corneocytes is supported in this study by the ${ }^{1} \mathrm{H}-\mathrm{NMR}$ spectra showing that: (1) SC samples exhibit a peak that is consistent with the ${ }^{1} \mathrm{H}$ spectrum for bulk water and keratin, which increases in area with increasing water content, and (2) linewidth of this peak is narrower for hydrated samples than desiccated sample, indicating that increasing water content allows increased motion of the molecules (primarily keratin protein) within the corneocytes. The absence of any hydration effect on the ${ }^{19} \mathrm{~F}$ spectra or 
$T_{1}$ for TFMB, which is located almost entirely in the lipids, is further evidence that water is located mainly outside the SC lipids.

Orientation of the SC samples within the external magnetic field significantly affected the ${ }^{19} \mathrm{~F}$-TFMB spectra. In the parallel orientation, the peak associated with TFMB in the lipids is sharp and unaffected by lipid extraction except for decreased area corresponding to decreased mass of TFMB. In the perpendicular orientation, the position of the TFMB peak is unchanged, but the linewidth has broadened, most significantly for the untreated sample. To identify the cause of the different linewidths for the perpendicular and parallel oriented samples will require further study. Measurements of extracted and untreated SC samples at both low and high hydration are needed. The $T_{1}$ for ${ }^{19}$ F-TFMB decreased when lipids were extracted, but appear to be unaffected by orientation, although additional measurements of perpendicular samples with and without lipid extraction are needed to confirm this observation definitively.

\section{NOMENCLATURE}

\section{Roman symbols}

$C_{\text {delip }} \quad$ TFMB concentration in the hydrated delipidized SC; mass of TFMB per mass of dry delipidized SC.

$C_{D I} \quad$ TFMB mass per mass of deionized water

$C_{l i p} \quad$ TFMB mass in the extractable lipids per mass of extractable lipids

$C_{P B S} \quad$ TFMB mass per mass of phosphate buffered saline (PBS) solution

$C_{\text {untreat }}$ TFMB mass in the hydrated untreated SC per mass of dry untreated SC

$f_{\text {lip }} \quad$ mass fraction of extractable lipids in the dry SC; mass ratio of the extracted lipids to the dry SC mass 
$f_{\text {lip,wet }} \quad$ mass fraction of extractable lipids in fully hydrated (wet) SC; mass ratio of the extracted lipids to the hydrated (wet) SC mass

$f_{T F M B, l i p}$ fraction of the sorbed TFMB in the untreated SC that is sorbed into the extractable lipid fraction

$K_{j / w} \quad$ partition coefficient for TFMB in $j$ relative to water; mass of TFMB in hydrated $j$ per volume of hydrated $j$ divided by the TFMB concentration (mass per volume) in the adjacent solution, where $j=\operatorname{cor}$ (for corneocytes), lip (for lipids), or SC

$K_{o / w} \quad$ octanol-water partition coefficient

$M_{z} \quad$ recovery magnetization response to the free induction pulse sequence to determine $T_{1}$

$M_{0} \quad$ equilibrium magnetization in the free induction pulse sequence to determine $T_{1}$

$n \quad$ number of samples or subjects

$P C_{\text {pro/w }}$ TFMB partition coefficient between the predominantly proteinaceous nonaqueous component of the corneocytes (i.e., proteins and the cell envelope and its covalently bound lipids in the dry delipidized SC) and the adjacent aqueous solution based on mass per mass concentrations

$T_{1} \quad$ spin-lattice relaxation time

$T_{2} \quad$ spin-spin relaxation time

$u_{c o r} \quad$ mass ratio of water in the hydrated corneocytes to the mass of dry corneocytes (i.e., the mass of dry delipidized SC).

$w_{w} \quad$ mass fraction of water per mass of hydrated SC

\section{Greek symbols}

$\rho_{j} \quad$ density of phase $j$; where $j=$ lip (for lipids), pro (for the proteinaceous dry delipidized $\mathrm{SC}$ ), $S C$ (for dry SC), or $w$ (for water) 
$\rho_{S C, d r y / w e t}$ mass of dry SC per unit volume of hydrated (wet) SC

$\rho_{c o r, d r y / w e t}$ mass of dry corneocytes (i.e., dry delipidized SC) per unit volume of hydrated (wet)

$\tau \quad$ evolution time of the recovery magnetization response to the free induction pulse sequence to determine $T_{1}$

$\varphi_{w} \quad$ volume fraction of water per volume of hydrated SC

\section{ACKNOWLEDGMENTS}

The authors acknowledge support from the National Science Foundation under Award Number 0854343. Essential support for the NMR facilities at the Colorado School of Mines that were used in this project was provided by the National Science Foundation through the Major Research Instrumentation Award Number 0923537 and a Cooperative Agreement through the MRSEC program in the Division of Materials Research (DMR-0820518). Discussions with P. Wertz from the University of Iowa are gratefully acknowledged. 


\section{REFERENCES}

1. Ya-Xian Z, Suetake T, Tagami H. 1999. Number of cell layers of the stratum corneum in normal skin - relationship to the anatomical location on the body, age, sex and physical parameters. Arch Dermatol Res 291(10):555-559.

2. Bouwstra JA, Gooris GS, van der Spek JA, Bras W. 1991. Structural investigations of human stratum corneum by small-angle X-ray scattering. J Invest Dermatol 97(6):10051012.

3. van Smeden J, Janssens M, Gooris GS, Bouwstra JA. 2014. The important role of stratum corneum lipids for the cutaneous barrier function. Biochim Biophys Acta-Lipids:295-313.

4. Bouwstra JA, Ponec M. 2006. The skin barrier in healthy and diseased state. Biochim Biophys Acta 1758(12):2080-2095.

5. Johnson ME, Blankschtein D, Langer R. 1997. Evaluation of solute permeation through the stratum corneum: Lateral bilayer diffusion as the primary transport mechanism. J Pharm Sci $86: 1162-1172$.

6. Frasch H, Barbero AM. 2003. Steady-state flux and lag time in the stratum corneum lipid pathway: Results from finite element models. J Pharm Sci 92:2196-2207.

7. Hadgraft J. 2004. Skin deep. Eur J Pharm Biopharm 58(2):291-299.

8. Wang TF, Kasting GB, Nitsche JM. 2006. A multiphase microscopic diffusion model for stratum corneum permeability. I. Formulation, solution, and illustrative results for representative compounds. J Pharm Sci 95(3):620-648.

9. Hansch C, Leo A, Hoekman D. 1995. Exploring QSAR: Hydrophobic, Electronic, and Steric Constants. Washington, DC: American Chemical Society. p 348. 
10. Wang TF, Kasting GB, Nitsche JM. 2007. A multiphase microscopic diffusion model for stratum corneum permeability. II. Estimation of physicochemical parameters, and application to a large permeability database. J Pharm Sci 96(11):3024-3051.

11. Kasting GB, Barai ND, Wang T-F, Nitsche JM. 2003. Mobility of water in human stratum corneum J Pharm Sci 92:2326-2340.

12. Li X, Johnson R, Weinstein B, Wilder E, Smith E, Kasting GB. 2015. Dynamics of water transport and swelling in human stratum corneum. Chem Eng Sci 138:164-172.

13. Fukushima E, Roeder SBW. 1981. Experimental Pulse NMR: A Nuts and Bolts Approach. Reading, MA, USA: Addison-Wesley, Advanced Book Program.

14. Syracuse Research Corporation (SRC). PhysProp Database, http://esc.syrres.com/fatepointer/search.asp, Last accessed July 13, 2016.

15. Kodiweera C, Yang Y, Bunge AL. Characterization of water self-diffusion in human stratum corneum using PFG-NMR. unpublished results.

16. Kodiweera C, Yang Y, Bunge AL. Characterization of self-diffusion of a model lipophilic compound in human stratum corneum using PFG-NMR. unpublished results.

17. Packer KJ, Sellwood TC. 1978. Proton magnetic resonance studies of hydrated stratum corneum. Part 2. Self-diffusion. J Chem Soc, Faraday Trans 2 74:1592-1606.

18. Packer KJ, Sellwood TC. 1978. Proton magnetic resonance studies of hydrated stratum corneum. Part 1. Spin lattice and transverse relaxation. J Chem Soc, Faraday Trans 2 $74: 1579-1591$.

19. Pieper J, Charalambopoulou G, Steriotis T, Vasenkov S, Desmedt A, Lechner RE. 2003. Water diffusion in fully hydrated porcine stratum corneum. Chem Phys 292:465-476. 
20. Foreman MI. 1976. A proton magnetic resonance study of water in human stratum corneum. Biochimica et Biophysica Acta (BBA) - General Subjects 437(2):599-603.

21. Silva CL, Topgaard D, Kocherbitov V, Sousa JJS, Pais A, Sparr E. 2007. Stratum corneum hydration: Phase transformations and mobility in stratum corneum, extracted lipids and isolated corneocytes. Biochim Biophys Act- Biomembranes 1768(11):2647-2659.

22. Hansen JR, Yellin W. 1972. NMR and infrared spectroscopic studies of stratum corneum hydration. In Jellinek HHG, editor Water Structure at the Water-Polymer Interface; Proceedings Symposium 161st National Meeting of the American Chemical Society, March 30-April 1, 1971, ed., New York, NY: Plenum Press. p 19-28.

23. Yamamura T, Tezuka T. 1989. The water-holding capacity of the stratum corneum measured by ${ }^{1} \mathrm{H}-\mathrm{NMR}$. J Invest Dermatol 93(1):160-164.

24. Vavasour I, Kitson N, MacKay A. 1998. What's water got to do with it? A nuclear magnetic resonance study of molecular motion in pig stratum corneum. J Invest Derm Symp P 3(2):101-104.

25. Laule C, Tahir S, Chia CLL, Vavasour IM, Kitson N, MacKay AL. 2010. A proton NMR study on the hydration of normal versus psoriatic stratum corneum: linking distinguishable reservoirs to anatomical structures. NMR Biomed 23(10):1181-1190.

26. Abraham W, Kitson N, Bloom M, Thewalt JL. 1997. Investigation of membrane structure and dynamics by deuterium NMR: Application to the stratum corneum. In Potts RO, Guy RH, editors. Mechanisms of Transdermal Drug Delivery, New York: Marcel Dekker, Inc. p 163-198. 
27. Bjorklund S, Andersson JM, Pham QD, Nowacka A, Topgaard D, Sparr E. 2014. Stratum corneum molecular mobility in the presence of natural moisturizers. Soft Matter 10(25):4535-4546.

28. Bjorklund S, Nowacka A, Bouwstra JA, Sparr E, Topgaard D. 2013. Characterization of stratum corneum molecular dynamics by natural-abundance C-13 solid-state NMR. Plos One 8(4):e61889.

29. Groen D, Gooris GS, Bouwstra JA. 2010. Model membranes prepared with ceramide EOS, cholesterol and free fatty acids form a unique lamellar phase. Langmuir 26(6):4168-4175.

30. Groen D, Gooris GS, Ponec M, Bouwstra JA. 2008. Two new methods for preparing a unique stratum corneum substitute. Biochim Biophys Act- Biomembranes 1778(10):24212429.

31. Bouwstra JA, de Graaff A, Gooris GS, Nijsse J, Wiechers JW, van Aelst AC. 2003. Water distribution and related morphology in human stratum corneum at different hydration levels. J Invest Dermatol 120:750-758.

32. Bouwstra JA, Gooris GS, Salomons-de Vries MA, van der Spek JA, Bras W. 1992. Structure of human stratum corneum as a function of temperature and hydration: a wideangle x-ray diffraction study. Int J Pharm 84(3):205-216.

33. Foreman MI, Bladon P, Pelling P. 1979. Proton NMR studies of human stratum corneum. Bioengineering and the Skin 2:48-58.

34. Raykar PV, Fung MC, Anderson BD. 1988. The role of protein and lipid domains in the uptake of solutes by human stratum corneum. Pharm Res 5(3):140-150.

35. Hansen S, Selzer D, Schaefer UF, Kasting GB. 2011. An extended database of keratin binding. J Pharm Sci 100(5):1712-1726. 
36. Blank IH, Moloney J, Emslie AG, Simon I, Apt C. 1984. The diffusion of water across the stratum corneum as a function of its water content. J Invest Dermatol 82(2):188-194.

37. Callaghan PT. 1991. Principles of Nuclear Magnetic Resonance Microscopy. ed., Oxford: Claredon Press, Oxford, 1991.

38. Romonchuk WJ, Bunge AL. 2010. Mechanism of enhanced dermal permeation of 4cyanophenol and methyl paraben from saturated aqueous solutions containing both solutes. Skin Pharmacol Physiol 23:152-163.

39. Swartzendruber DC, Wertz PW, Madison KC, Downing DT. 1987. Evidence that the corneocyte has a chemically bound lipid envelope. J Invest Dermatol 88(6):709-713.

40. Nemes Z, Steinert PM. 1999. Bricks and mortar of the epidermal barrier. Exp Mol Med 31:5-19.

41. Pappinen S, Hermansson M, Kuntsche J, Somerharju P, Wertz P, Urtti A, Suhonen M. 2008. Comparison of rat epidermal keratinocyte organotypic culture (ROC) with intact human skin: Lipid composition and thermal phase behavior of the stratum corneum. Biochim Biophys Act- Biomembranes 1778(4):824-834.

42. Elias PM, Cooper ER, Korc A, Brown BE. 1981. Percutaneous transport in relation to stratum corneum structure and lipid composition. J Invest Dermatol 76(4):297-301.

43. Nitsche JM, Wang T-F, Kasting GB. 2006. A two-phase analysis of solute partitioning into the stratum corneum. J Pharm Sci 95:649-666.

44. Gay CL, Guy RH, Golden GM, Mak VHW, Francoeur ML. 1994. Characterization of lowtemperature (i.e., $<65^{\circ} \mathrm{C}$ ) lipid transitions in human stratum corneum. J Invest Dermatol 103(2):233-239. 
45. Van Duzee BF. 1975. Thermal analysis of human stratum corneum. J Invest Dermatol 65(4):404-408.

46. Lampe MA, Burlingame AL, Whitney J, Williams ML, Borwn BE, Roitman E, Elias PM. 1983. Human stratum corneum lipids: characterization and regional variations. J Lipid Res $24: 120-130$.

47. Cleek RL, Bunge AL. 1993. A new method for estimating dermal absorption from chemical exposure. 1. General approach. Pharm Res 10(4):497-506.

48. Bunge AL, Cleek RL. 1995. A new method for estimating dermal absorption from chemical exposure. 2. Effect of molecular weight and octanol-water partitioning. Pharm Res 12(1):8794.

49. Roberts MS, Pugh WJ, Hadgraft J. 1996. Epidermal permeability-penetrant structure relationships. 2: The effect of $\mathrm{H}$-bonding groups in penentrants on their diffusion through the stratum corneum. Int J Pharm 132:23-32.

50. Pugh WJ, Roberts MS, Hadgraft J. 1996. Epidermal permeability-penetrant structure relationships. 3: The effect of hydrogen-bonding interactions and molecular size on diffusion across the stratum corneum. Int J Pharm 138:149-165.

51. Vecchia BE, Bunge AL. 2002. Partitioning of chemicals into skin: Results and prediction. In Hadgraft J, Guy RH, editors. Transdermal Drug Delivery, 2nd ed., New York: Marcel Dekker. p 143-198.

52. Wang LM, Chen LJ, Lian GP, Han LJ. 2010. Determination of partition and binding properties of solutes to stratum corneum. Int J Pharm 398(1-2):114-122.

53. Hansen S, Lehr C-M, Schaefer UF. 2013. Improved input parameters for diffusion models of skin absorption. Adv Drug Deliv Rev 65(2):251-264. 
54. Rice RH, Xia Y, Alvarado RJ, Phinney BS. 2010. Proteomic Analysis of Human Nail Plate. J Proteome Res 9(12):6752-6758.

55. Weishaupt D, Köchli VD, Marincek B. 2006. How does MRI work?: An introduction to the physics and function of magnetic resonance imaging. Second ed., New York: Springer. p 169.

56. Pilgram GSK, Vissers DCJ, van der Meulen H, Pavel S, Lavrijsen AP, Bouwstra JA, Koerten HK. 2001. Aberrant lipid organization in stratum corneum of patients with atopic dermatitis and lamellar ichthyosis. J Invest Dermatol 117:710-717.

57. Al-Amoudi A, Dubochet J, Norlen L. 2005. Nanostructure of the epidermal extracellular space as observed by cryo-electron microscopy of vitreous sections of human skin. J Invest Dermatol 124(4):764-777.

58. Van den Bergh BAI, Swartzendruber DC, Bos-van der Geest A, Hoogstraate JJ, Schrijvers AHGJ, Boddé HE, Junginger HE, Bouwstra JA. 1997. Development of an optimal protocol for the ultrastructural examination of skin by transmission electron microscopy. J Microsc 187(2):125-133.

59. Detrimental effects of low sample volume on NMR signal, http://nmr.chem.ucsb.edu/protocols/sample volume.html, Last accessed July 13, 2016. 


\section{FIGURE LEGENDS}

Figure 1. TFMB (2-(trifluoromethyl) benzonitrile); $\mathrm{CF}_{3} \mathrm{C}_{6} \mathrm{H}_{4} \mathrm{CN}$, CAS No. 447-60-9; Molecular weight $=171.1 ;$ Melting point $=7.5^{\circ} \mathrm{C} ;$ Specific gravity $=1.29 ; \log K_{o / w}($ estimated $)=2.50^{9}$

Figure 2. NMR experimental methods: (a) sample packing in the NMR tube for orienting the SC layers perpendicular and parallel to the direction of the external magnetic field; (b) single pulse sequence (not drawn to scale) to obtain spectrum; and (c) Inversion recovery pulse sequence (not drawn to scale) to determine $T_{1}$ from the observed magnetization recovery over the evolution time $(\tau)$.

Figure 3. ${ }^{1} \mathrm{H}$ spectra measured relative to the DI water reference: (a) determined in hydrated $\left(\varphi_{w}\right.$ $=0.84$ ) and desiccated SC samples from one subject with or without lipid extraction (normalized to the maximum amplitude) and compared with water; and (b) determined in fingernail clippings measured desiccated (dashed) and hydrated (solid) compared with water.

Figure 4. ${ }^{19}$ F-TFMB spectra (normalized to the maximum observation) for highly hydrated SC with lipids and with lipids extracted $\left(\varphi_{w}=0.80\right.$ and 0.77 , respectively) and oriented parallel $(\|)$ to the external magnetic field direction compared with spectra for TFMB in water, 1-octanol and neat TFMB.

Figure 5. ${ }^{19}$ F-TFMB spectra (normalized to the maximum observation) for SC oriented parallel $(\|)$ or perpendicular $(\perp)$ to the external magnetic field direction determined in samples at low $\left(0.5<\varphi_{w}<0.6\right)$ and high $\left(\varphi_{w}=0.80\right)$ hydration and with lipids or with lipids extracted. Note that spectrum B is spectrum A in Figure 4.

Figure 6. $T_{1}$ values determined at $25^{\circ} \mathrm{C}$ for ${ }^{19} \mathrm{~F}$-TFMB in SC oriented either parallel (\|l) or perpendicular $(\perp)$ to the external magnetic field and with or without lipids extracted: (a) plotted as a function of the volume fraction of water; and (b) compared with TFMB in itself, 1-octanol, and water. Individual measurements are indicated as symbols. The bar height corresponds to the average of all measurements, excluding the two outliers designated as $\mathrm{X}$. 
Table 1. Mass fraction of lipids extracted from dry human SC samples

\begin{tabular}{|c|c|c|c|}
\hline Subject ID & $\begin{array}{l}\text { Sorption Experime } \\
f_{\text {lip }}\end{array}$ & $s^{a}$ & $\begin{array}{l}\text { NMR Experiments } \\
f_{\text {lip }}\end{array}$ \\
\hline AR & & & 0.361 \\
\hline $\mathrm{AV}$ & $0.228 \pm 0.033$ & 6 & 0.237 \\
\hline $\mathrm{AW}^{b}$ & $0.195 \pm 0.018$ & 6 & 0.203 \\
\hline $\mathrm{AW}^{b}$ & $0.201 \pm 0.004$ & 5 & \\
\hline $\mathrm{AY}$ & & & 0.260 \\
\hline BB & $0.274 \pm 0.011$ & 5 & \\
\hline $\mathrm{BC}$ & $0.223 \pm 0.017$ & 6 & \\
\hline $\mathrm{BD}^{c}$ & $0.140 \pm 0.019$ & 5 & 0.217 \\
\hline $\mathrm{BD}^{c}$ & & & 0.224 \\
\hline $\mathrm{BD}^{c}$ & & & 0.194 \\
\hline BG & & & 0.303 \\
\hline $\mathrm{BH}$ & & & 0.256 \\
\hline BI & & & 0.293 \\
\hline $\begin{array}{l}\text { All Subjects } \\
n \text { total }\end{array}$ & $\begin{array}{r}0.210 \pm 0.044^{d} \\
33\end{array}$ & & $\begin{array}{l}0.255 \pm 0.052^{e} \\
10\end{array}$ \\
\hline
\end{tabular}

${ }^{a}$ Mean \pm one standard deviation; $n=$ number of pieces of skin evaluated

${ }^{b}$ About 7 months between replicate measurements of SC from subject AW.

${ }^{c}$ 2-3 months between replicate measurements of SC from subject BD.

${ }^{d}$ Calculated from the average values of 6 subjects (i.e., replicate of subject AW was treated as a separate subject). Equals $0.213 \pm 0.049$ if replicate of subject AW is not treated as a separate subject (i.e., averaged over 5 subjects using the average of replicate measurements from subject AW $=0.198$ ); equals $0.210 \pm 0.043$ if calculated for all 33 measurements treated separately.

${ }^{e}$ Calculated using 10 individual measurements; equal to $0.266 \pm 0.052$ if calculated for 8 subjects (average of triplicate measurements from subject $\mathrm{BD}=0.212$ ). 
Table 2. Equilibrium TFMB sorption at $32^{\circ} \mathrm{C}$ into fully hydrated isolated $\mathrm{SC}$ that was and was not treated to extract lipids normalized by the concentration of the adjacent solution.

\begin{tabular}{|c|c|c|}
\hline \multirow{2}{*}{$\begin{array}{l}\text { Quantity } \\
C_{\text {untreat }} / C_{D I}\end{array}$} & \multicolumn{2}{|c|}{ Results (mean \pm standard dev) ${ }^{a}$} \\
\hline & 57.5 & \pm 31.8 \\
\hline$C_{\text {untreat }} / C_{P B S}$ & 63.4 & \pm 31.4 \\
\hline$C_{\text {delip }} / C_{P B S}$ & 3.97 & \pm 2.12 \\
\hline$C_{l i p} / C_{P B S}$ & 287 & \pm 120 \\
\hline$f_{T F M B, l i p}$ & 0.95 & \pm 0.02 \\
\hline$P C_{p r o / w}$ & $0.91^{b}$ & \\
\hline \multirow[t]{2}{*}{$K_{s c / w}$} & 19.7 & \pm 9.7 for $f_{l i p}=0.21$ \\
\hline & 18.7 & \pm 9.3 for $f_{\text {lip }}=0.15$ \\
\hline$K_{l i p / w}$ & 258 & \pm 108 \\
\hline$K_{c o r / w}$ & 1.05 & \pm 0.56 \\
\hline$K_{\text {lip/cor }}=K_{\text {lip/w }} / K_{\text {corl } / w}$ & 246 & $\pm 167^{c}$ \\
\hline \multicolumn{3}{|c|}{$\begin{array}{l}\text { Mean } \pm \text { one standard deviation of the mean from each of } \\
\text { six separate experiments consisting of measurements in } 5 \\
\text { or } 6 \text { pieces of SC from five different subjects with one } \\
\text { subject replicated. } \\
b \text { Difference of the average value of } C_{d e l i p} / C_{P B S} \text { and } u_{c o r}= \\
3.06 \text { as specified in Eq. }(5) \text {. } \\
{ }^{c} \text { Ratio calculated from the average values for } K_{l i p / P B S} \text { and } \\
K_{\text {corlPBS; standard deviation by propagation of error }} \\
\text { analysis assuming zero covariance. }\end{array}$} \\
\hline
\end{tabular}


Table 3. Predictions of partition coefficients for SC and its components calculated using $K_{o / w}$ in published equations $^{a}$

\begin{tabular}{|c|c|c|c|c|}
\hline Eq. No. & $\boldsymbol{K}_{S C / w}$ & $\boldsymbol{K}_{l i p / w}$ & $P C_{p r o / w}$ & $\boldsymbol{K}_{c o r / w}$ \\
\hline $\mathrm{T} 1$ & 70.8 & & & \\
\hline $\mathrm{T} 2$ & 28.2 & & & \\
\hline $\mathrm{T} 3$ & 10.6 & & & \\
\hline $\mathrm{T} 4$ & 10.3 & & & \\
\hline T5, T8, T9 & $10.3,9.4^{b}$ & 45.5 & 25.6 & 7.6 \\
\hline T6, T8, T9 & $10.3,9.4^{b}$ & 47.8 & 25.0 & 7.4 \\
\hline T7, T8, T9 & $13.1,12.0^{b}$ & 56.3 & 33.8 & 9.7 \\
\hline Eq. No. & \multicolumn{3}{|l|}{ Equation } & Source \\
\hline $\mathrm{T} 1$ & \multicolumn{3}{|c|}{$\log _{10} K_{S C / w}=0.74 \log _{10} K_{o / w}$} & 47,48 \\
\hline $\mathrm{T} 2$ & \multicolumn{3}{|c|}{$\log _{10} K_{S C / w}=-0.024+0.59 \log _{10} K_{o / w}$} & 49,50 \\
\hline $\mathrm{T} 3$ & \multicolumn{3}{|c|}{$\log _{10} K_{S C / w}=-0.059+0.434 \log _{10} K_{o / w}$} & 51 \\
\hline $\mathrm{T} 4$ & \multicolumn{3}{|c|}{$\log _{10} K_{S C / w}=-0.290+0.432\left(\log _{10} K_{o / w}+0.5\right)$} & 51 \\
\hline T5a & \multicolumn{3}{|c|}{$\log _{10} K_{l i p / w}=-0.37+0.81 \log _{10} K_{o / w}$} & 43 \\
\hline $\mathrm{b}$ & \multicolumn{3}{|c|}{$\log _{10} P C_{p r o / w}=0.73+0.27 \log _{10} K_{o / w}$} & 43 \\
\hline T6a & \multicolumn{3}{|c|}{$\log _{10} K_{l i p / w}=-0.046+0.69 \log _{10} K_{o / w}$} & 51 \\
\hline $\mathrm{b}$ & \multicolumn{3}{|c|}{$\log _{10} P C_{p r o / w}=0.62+0.31 \log _{10} K_{o / w}$} & 52 \\
\hline $\mathrm{T} 7 \mathrm{a}$ & \multicolumn{3}{|c|}{$\log _{10} K_{l i p / w}=0.075+0.671 \log _{10} K_{o / w}$} & 53 \\
\hline $\mathrm{b}$ & \multicolumn{3}{|c|}{$\log _{10} P C_{p r o / w}=0.73+0.32 \log _{10} K_{o / w}$} & 53 \\
\hline
\end{tabular}

Definitions

$\mathrm{T} 8$

$$
\begin{array}{ll}
K_{c o r / w}=\left(P C_{p r o / w}+u_{c o r}\right) \frac{\rho_{c o r, d r y / w e t}}{\rho_{w}} & \text { Eqs. (5) \& (8) } \\
K_{S C / w}=\varphi_{l i p} K_{l i p / w}+\left(1-\varphi_{\text {lip }}\right) K_{c o r / w} & 43,52,53
\end{array}
$$

where $\varphi_{\text {lip }}=$ lipid volume fraction of hydrated SC,

$$
\varphi_{\text {lip }}=f_{\text {lip }} \rho_{S C, \text { dry/wet }} / \rho_{\text {lip }}
$$

\footnotetext{
${ }^{a}$ Input parameter values: $\log K_{o / w}$ (estimated, experimental value not available) $=2.5 ; u_{c o r}$ $=3.06, \rho_{l i p}=0.9 \mathrm{~g} / \mathrm{mL}, \rho_{c o r, d r y / w e t}=0.26 \mathrm{~g} / \mathrm{mL}$; for $f_{l i p}=0.21, \varphi_{l i p}=0.072, \rho_{S C, d r y / \text { wet }}=$ $0.310 \mathrm{~g} / \mathrm{mL}$; for $f_{\text {lip }}=0.15, \varphi_{\text {lip }}=0.049, \rho_{S C, d r y / \text { wet }}=0.295 \mathrm{~g} / \mathrm{mL}$

${ }^{b}$ Calculated assuming $f_{\text {lip }}=0.21, f_{\text {lip }}=0.15$
} 


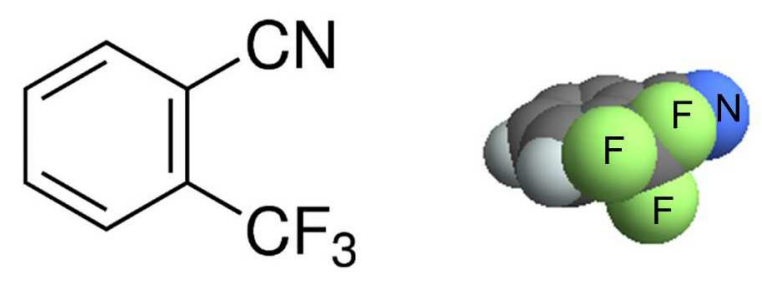


(a)

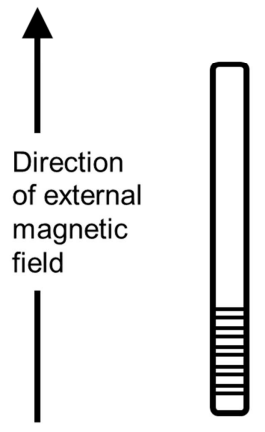

SC layers packed perpendicular to the external magnetic field

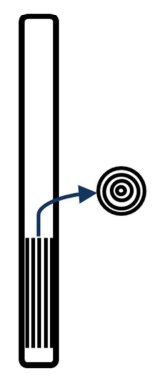

SC layers packed parallel to the external magnetic field

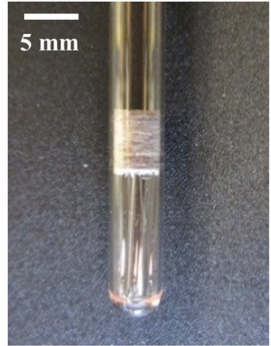

SC circles stacked perpendicular to the external magnetic field

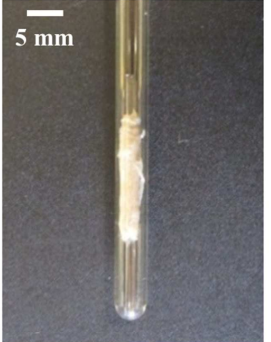

$\mathrm{SC}$ sheet rolled on a glass pipette tip parallel to the external magnetic field (b)

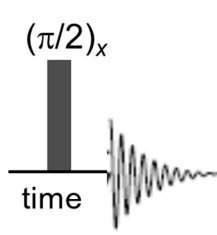

Spectrum

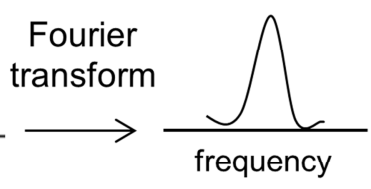

(c)

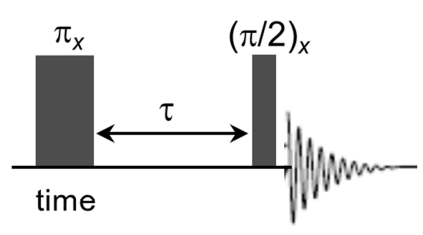

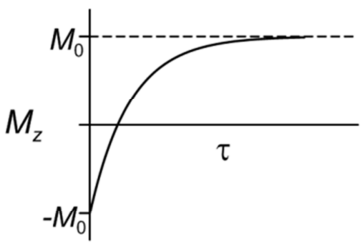


(a)

${ }^{1} \mathrm{H}$ Spectra

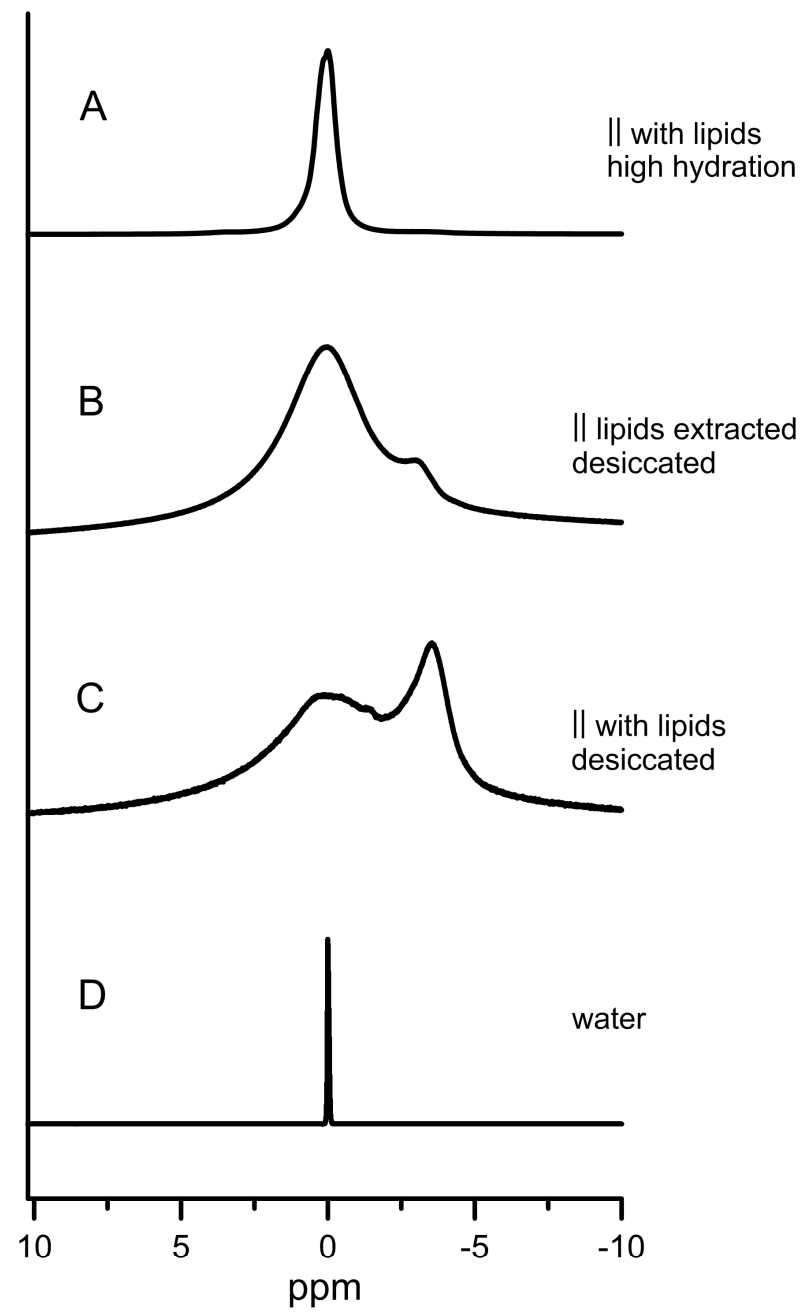

(b) ${ }^{1} \mathrm{H}$ Spectra

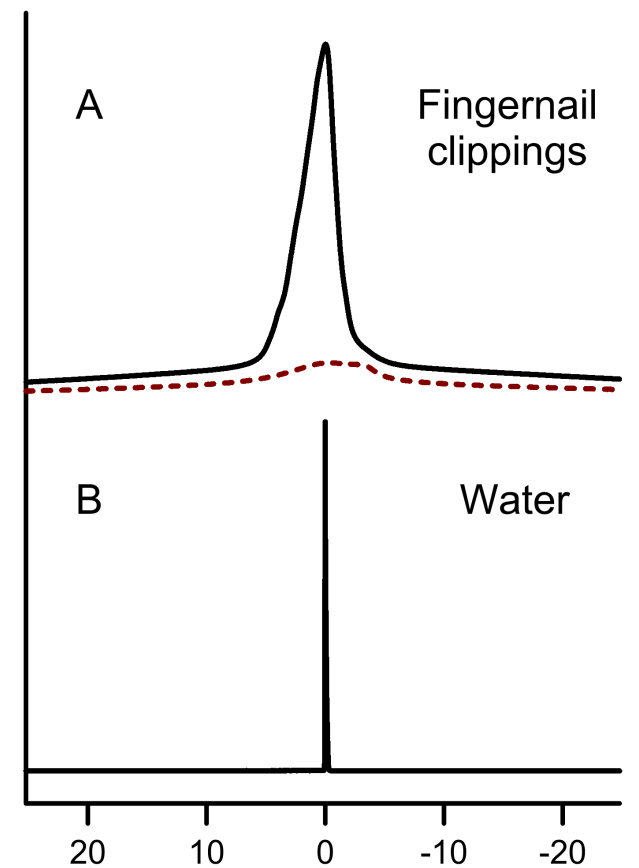


${ }^{19} \mathrm{~F}$ Spectra

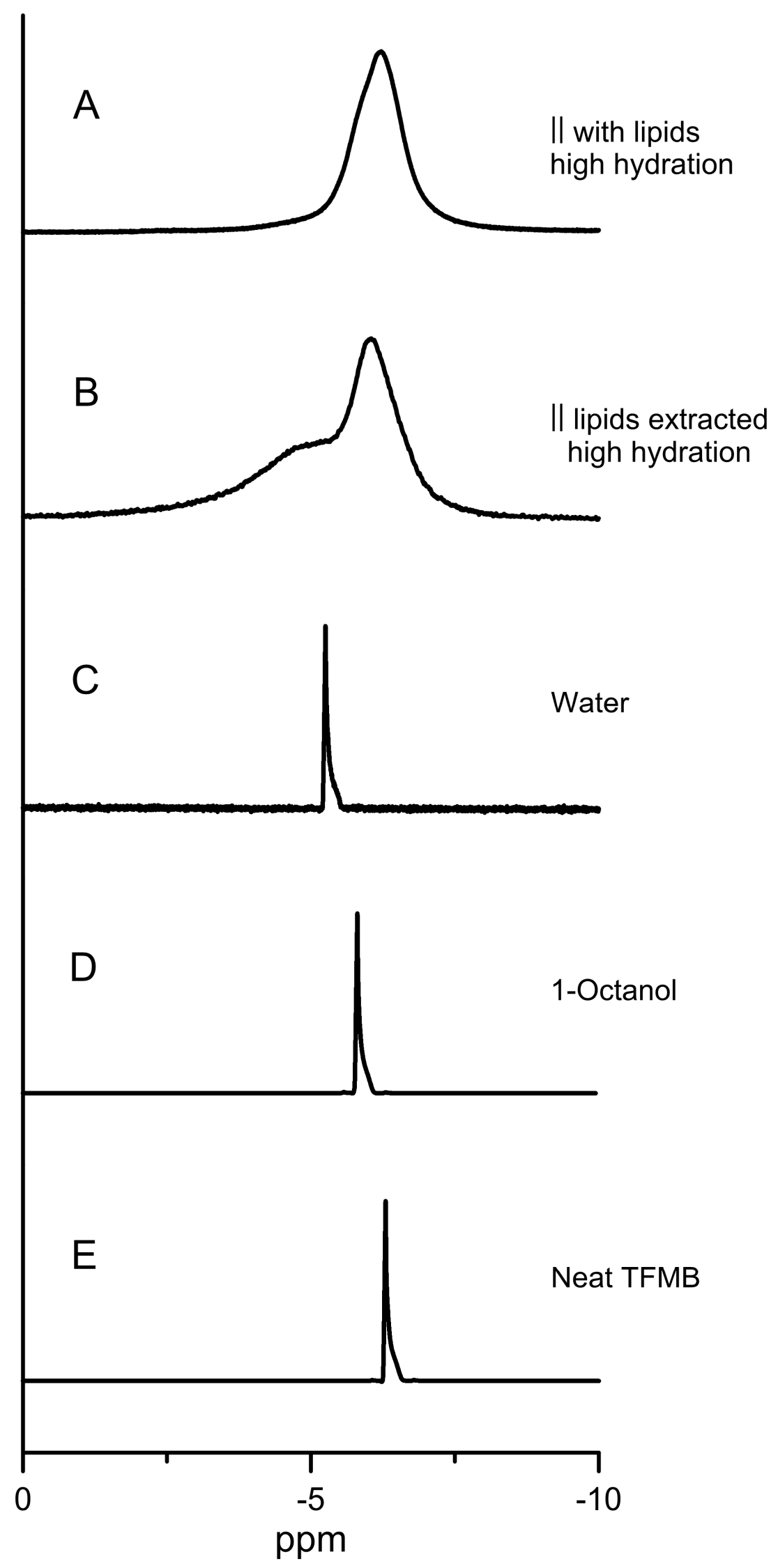


${ }^{19} \mathrm{~F}$ Spectra
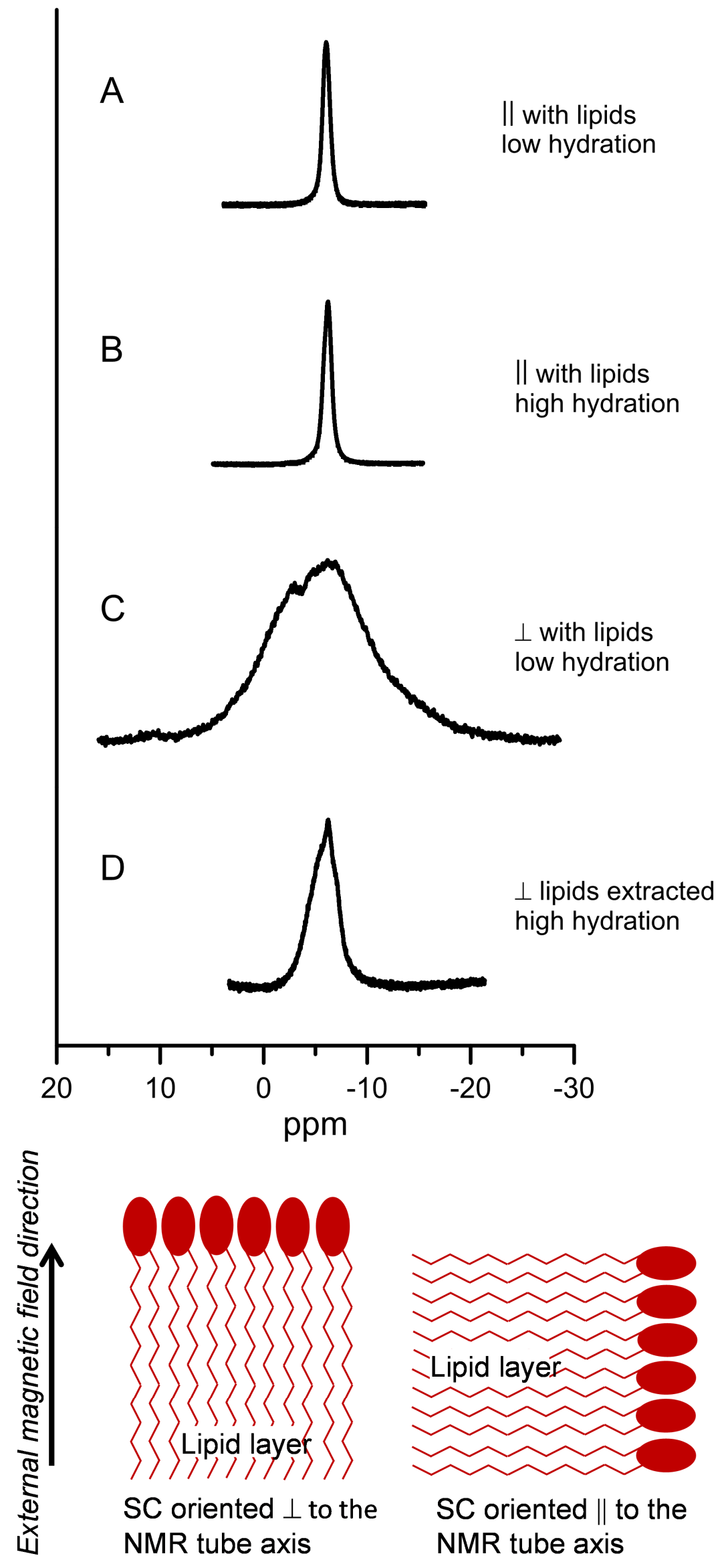
(a)

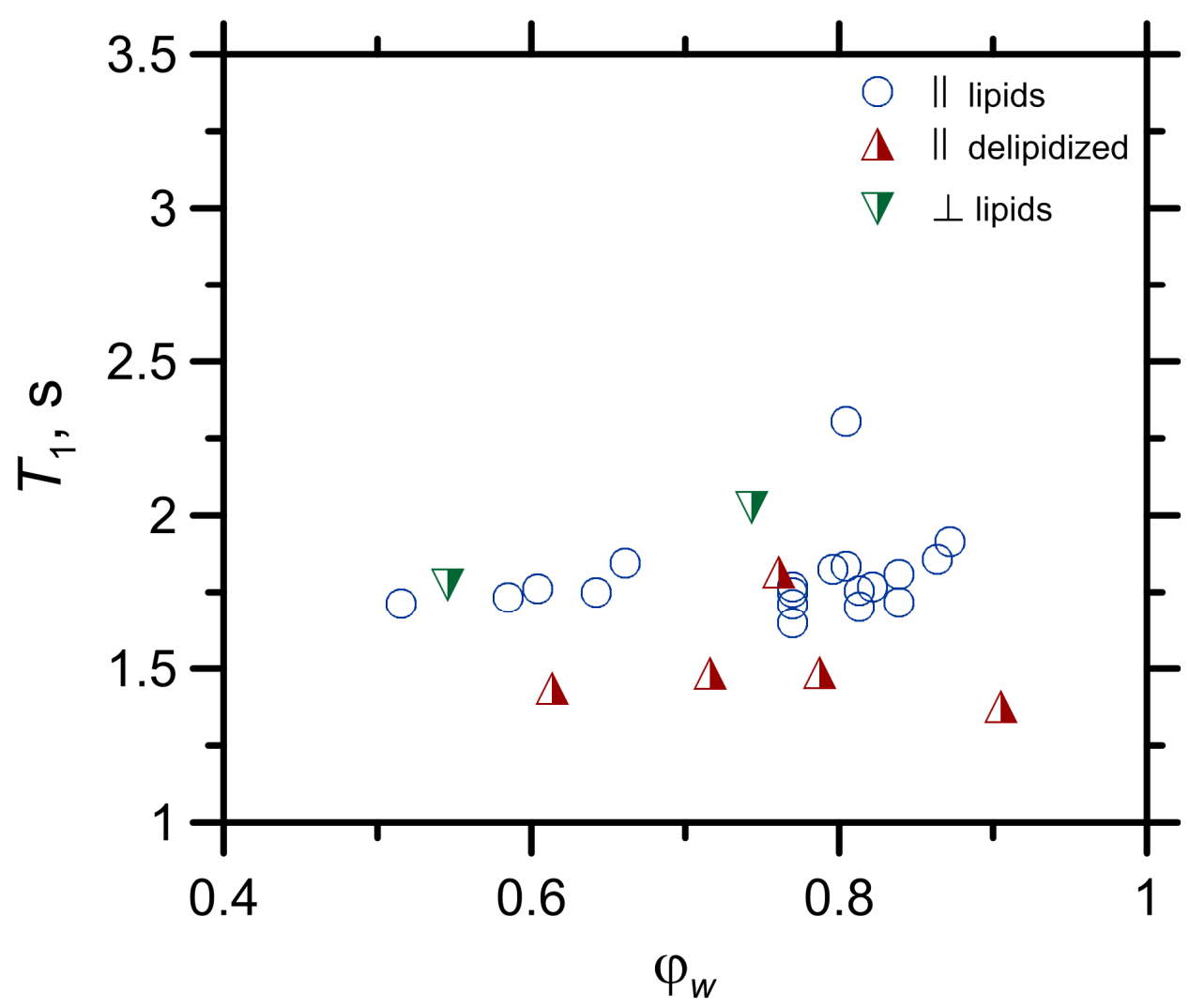

(b)

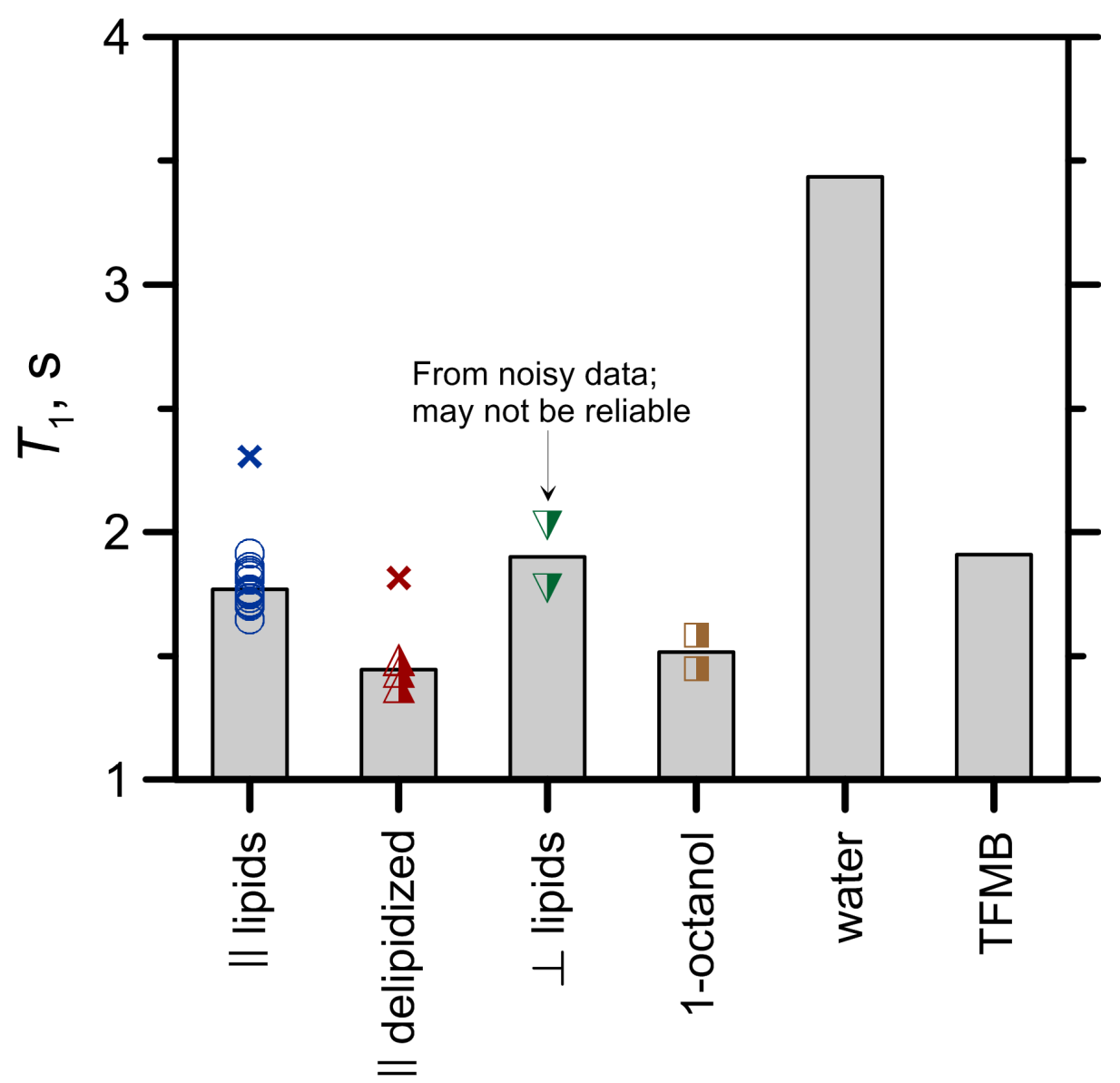

